$$
\{[f(x+(D-1) \theta)-f(x-\theta D)] F(y)\}_{y=0}=\theta \frac{d f(x)}{d x} .
$$

Blissard's remark, "An equation which has a representative quantity is not susceptible to any algebraic operation by which the indices would be affected," becomes

$$
(D f)^{2} \neq D^{2} f \text {. }
$$

UNIVERSITY OF WASHINGTON

\title{
ON FOURTH ORDER SELF-ADJOINT DIFFERENCE SYSTEMS*
}

BY V. V. LATSHAW

A linear difference expression for which the differential transform is self-adjoint (anti-self-adjoint) we shall call self-adjoint (anti-self-adjoint). $\dagger$ We choose two fourth order difference equations

$$
\begin{aligned}
L^{+}(u) & \equiv p(x)[u(x+2)+u(x-2)] \\
& +\lambda[u(x+1)+u(x-1)]+R(x) u(x)=0, \\
L^{-}(u) & \equiv p(x)[u(x+2)-u(x-2)] \\
& +\lambda[u(x+1)-u(x-1)]=0,
\end{aligned}
$$

where $L^{+}(u)$ is self-adjoint and $L^{-}(u)$ anti-self-adjoint for the range $(x=a, a+1, \cdots, b-1 ; b-a \geqq 4) . R(x)$ and $p(x)$ are both real, $p(x)$ being a non-vanishing periodic function of period two; $\lambda$ is a parameter.

Let the functions $\left(y_{1}, y_{2}, y_{3}, y_{4}\right)$ constitute a fundamental set of solutions for either (1) or (2), and $\left(w_{1}, w_{2}, w_{3}, w_{4}\right)$ the set adjoint to it. The two sets are related by the equations

* Presented to the Society, October 30, 1937.

$\dagger \mathrm{J}$. Kaucky, Sur les équations aux différences finies qui sont identiques d leurs adjointes, Publications of the Faculty of Sciences, University of Masaryk, No. 22 (1922). For a discussion of adjoint differential expressions of infinite order, see H. T. Davis, The Theory of Linear Operators, 1936, pp. 474-475. 


$$
\begin{aligned}
& w_{j}(x) p(x)=\frac{A_{4 j}(x+1)}{A(x+1)},
\end{aligned}
$$

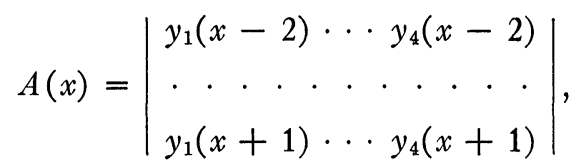

in which $A(x)$ denotes the Casorati determinant and $A_{i j}(x)$ the cofactors of its elements. Since $w_{j}$ satisfies the equation $L(u)=0$, one can write

$$
w_{i}=c_{i 1} y_{1}(x)+\cdots+c_{i 4} y_{4}(x), \quad(i=1,2,3,4) .
$$

The $c_{i j}$ have properties stated in the following theorem.*

Theorem 1. The matrix $C^{+}$of the substitution (4) is skew-symmetric and $C^{-}$symmetric. Further, there is a set of relations involving $c_{i j}{ }^{+}$and the second order minors of $A^{+}(x)$ from which the $c_{i j}{ }^{+}$ may be calculated explicitly:

$$
\begin{gathered}
\sum_{j>i=1}^{4} c_{i j}{ }^{+}\left(\begin{array}{rr}
y_{i} & y_{j} \\
-2 & -1
\end{array}\right)^{-}=0, \quad \sum c_{i j}+\left(\begin{array}{cc}
y_{i} & y_{j} \\
-2 & 0
\end{array}\right)^{-}=-\frac{1}{p(x)} \\
\sum c_{i j}{ }^{+}\left(\begin{array}{rr}
y_{i} & y_{j} \\
-2 & 1
\end{array}\right)^{-}=\frac{\lambda}{p(x) p(x+1)} \\
\sum_{j>i=1}^{4} c_{i j}^{+}\left(\begin{array}{rr}
y_{i} & y_{j} \\
-1 & 0
\end{array}\right)^{-}=0, \quad \sum c_{i j}^{+}\left(\begin{array}{cc}
y_{i} & y_{j} \\
-1 & 1
\end{array}\right)^{-}=-\frac{1}{p(x+1)} \\
\sum c_{i j}+\left(\begin{array}{rr}
y_{i} & y_{j} \\
0 & 1
\end{array}\right)^{-}=0 .
\end{gathered}
$$

We resolve (4) to get

$$
c_{i j}{ }^{+}=\left[w_{i}(x-2) A_{1 j}(x)+\cdots+w_{i}(x+1) A_{4 j}(x)\right] \frac{1}{A(x)} .
$$

We use $(1,2,3)$ to furnish the following relations

* We introduce a $(+)$ and $(-)$ convention to distinguish between quantities associated with (1) and (2) respectively. Also we set

$$
u(x+r) v(x+s) \pm v(x+r) u(x+s)=\left(\begin{array}{ll}
u & v \\
r & s
\end{array}\right)^{ \pm} .
$$


(6)

$$
\begin{aligned}
w_{j}(x-1) p(x-1) & =\frac{A_{4 j}(x)}{A(x)}, \\
w_{j}(x-2) p(x)+\lambda w_{j}(x-1) & =\frac{A_{3 j}(x)}{A(x)}, \\
\pm w_{j}^{ \pm}(x) p(x) & =\frac{A_{1 j}(x)}{A^{ \pm}(x)}, \\
\mp\left[\lambda w_{j}^{ \pm}(x)+p(x+1) w_{j}^{ \pm}(x+1)\right] & =\frac{A_{2 j}(x)}{A^{ \pm}(x)} .
\end{aligned}
$$

Combining (5) and (6) we find

$$
\begin{aligned}
c_{i j}^{ \pm}=p(x+1)\left(\begin{array}{cc}
w_{i}^{ \pm} & w_{j}^{ \pm} \\
1 & -1
\end{array}\right)^{\mp} & +p(x)\left(\begin{array}{cc}
w_{i}^{ \pm} & w_{j}^{ \pm} \\
0 & -2
\end{array}\right)^{\mp} \\
& +\lambda\left(\begin{array}{cc}
w_{i}^{ \pm} & w_{j}^{ \pm} \\
0 & -1
\end{array}\right)^{\mp} .
\end{aligned}
$$

Obviously we have $c_{i j}{ }^{+}=-c_{j i}{ }^{+}$and $c_{i j}=c_{j \bar{i}}$. Combine (3) and (4) to eliminate the $w$ 's. These equations together with $L^{+}\left(y_{i}^{+}\right)$ $=0$ yield the set of equations involving the minors. The sixth order determinant composed of the two-rowed minors is nonvanishing $\left(=\left[A^{+}(x)\right]^{3}\right)$.*

In the development of adjoint difference systems the formula

$$
\sum f(x+1)=f(x)+\sum f(x)
$$

is used to provide a Lagrange relation

$$
\sum_{x=a}^{b-1}[v L(u)-u L(v)]=\Pi(u, v)=U_{1} U_{8}+\cdots+U_{8} V_{1},
$$

in which the $U_{i}$ are eight linearly independent forms arbitrarily chosen :

$$
\begin{aligned}
U_{i}= & a_{i 1} u(a-2)+\cdots+a_{i 4} u(a+1) \\
& +b_{i 1} u(b-2)+\cdots+b_{i 4} u(b+1) .
\end{aligned}
$$

If the two systems

$$
\begin{array}{ll}
L(u)=0, & U_{1}=U_{2}=U_{3}=U_{4}=0, \\
L(v)=0, & V_{1}=V_{2}=V_{3}=V_{4}=0,
\end{array}
$$

* Turnbull, The Theory of Determinants, Matrices, and Invariants, 1929, p. 87. 
are equivalent, we shall call them self-adjoint difference systems.

THEOREM 2. Given the fourth order difference systems composea' of the equations

$$
L^{ \pm}(u)=0, \quad U_{i}=0, \quad(i=1,2,3,4),
$$

defined in (1), (2), (10), let $u(x)$ and $v(x)$ be any pair of functions satisfying $U_{i}=0$; then $\Pi(u, v) \equiv 0$ is a necessary and sufficient condition that the given system be self-adjoint.

That the above condition is necessary needs no proof. Let $\left(u_{1}, u_{2}, u_{3}, u_{4}\right)$ be four linearly independent functions satisfying $U_{i}=0$ and $u$ any linear combination of them. Through substitution the identity $\Pi(u, v) \equiv 0$ gives

$$
U_{5}\left(u_{i}\right) V_{4}(u)+\cdots+U_{8}\left(u_{i}\right) V_{1}(u)=0, \quad(i=1,2,3,4) .
$$

Since the set $\left(U_{1}, U_{2}, \cdots, U_{8}\right)$ is linearly independent, it follows that the four systems of constants comprising the coefficients of the $V$ 's are linearly independent and we have $V_{1}(u)=V_{2}(u)=V_{3}(u)=V_{4}(u)=0$. A similar argument shows that any function satisfying the given boundary conditions will also satisfy the adjoint boundary conditions.

We record some examples which fulfill the condition for selfadjointness.

$$
\begin{aligned}
U_{1^{ \pm}}^{ \pm}= & a_{11}^{ \pm} u(a-2)+a_{12} u(a-1)+p(a) u(a) \\
& +b_{13} u(b)+b_{14} u(b+1)=0, \\
U_{2^{ \pm}}= & \pm a_{12} u(a-2)+a_{22}^{ \pm} u(a-1)+\lambda u(a) \\
& +p(a+1) u(a+1)+b_{24} u(b+1)=0, \\
U_{3}^{ \pm}= & \pm b_{13} u(a-2) \pm p(b) u(b-2) \pm \lambda u(b-1) \\
& +b_{33}^{ \pm} u(b)+b_{34} u(b+1)=0, \\
U_{4}^{ \pm}= & \pm b_{14} u(a-2) \pm b_{24} u(a-1) \pm p(b+1) u(b-1) \\
& \pm b_{34} u(b)+b_{44}^{ \pm} u(b+1)=0,
\end{aligned}
$$

with the agreement that $a_{11}-=a_{22}-=b_{33}{ }^{-}=b_{44}-=0$. For self-adjoint Sturmian boundary conditions we make the added restrictions, $b_{13}=b_{14}=b_{24}=0$.

We now introduce a function $G^{ \pm}(x, t)$ defined for $(a-1<x<b)$, 
$(a \leqq t<b)$, satisfying the given boundary conditions and for which $L^{ \pm}\left[G^{ \pm}(i, j)\right]=\delta_{i j}$.

THeOREM 3. Let $\lambda_{1}{ }^{ \pm}, \lambda_{2}{ }^{ \pm}, \cdots$ be sets of characteristic values for the systems $L^{ \pm}(u)=0, U_{i}=0$ defined in (13). There exists a $\lambda_{k}{ }^{+}$ in the interval $\left(\lambda_{\bar{i}} \leqq \lambda_{k}{ }^{+}<\lambda_{i+1}^{-}\right)$provided

$G^{-}(b, b-1)-G^{+}(b, b-1)-\sum_{x=a}^{b-1}\left[G^{-}(x-1, x)+G^{+}(x-1, x)\right] \neq 0$,
$\left(\lambda_{\bar{i}}^{-} \leqq \lambda \leqq \lambda_{\bar{i}+1}\right)$.

If $(b-a)$ is an odd integer, then every value of $\lambda^{-}$is a characteristic value.

Let $D^{ \pm}(\lambda)=0$ be the characteristic equations for (13). By writing $D^{ \pm}(\lambda)$ in determinant form one finds

$$
\frac{d D^{ \pm}}{d \lambda}=2 D^{ \pm}\left[G^{ \pm}(b, b-1) \pm \sum_{x=a}^{b-1} G^{ \pm}(x-1, x)\right] .
$$

This relation enables us to write

$$
\begin{aligned}
\frac{d}{d \lambda}\left(\frac{D^{-}}{D^{+}}\right)=\frac{2 D^{-}}{D^{+}} & {\left[G^{-}(b, b-1)-G^{+}(b, b-1)\right.} \\
& \left.-\sum_{x=a}^{b-1}\left\{G^{-}(x-1, x)+G^{+}(x-1, x)\right\}\right] .
\end{aligned}
$$

Between the two real consecutive zeros $\lambda_{i}^{-}$and $\lambda_{i+1}^{-}$of $D^{-}(\lambda)$ either the bracketed expression or $D^{+}(\lambda)$ must vanish. By assumption the bracketed expression does not vanish.

The proof of the final statement in the theorem consists merely in noticing that $D^{-}(\lambda)$ for this case is a skew-symmetric determinant of odd order.

LEHIGH UNIVERSITY 